\title{
Expression phénotypique de la polypose adénomateuse familiale
}

Il est bien connu en génétique médicale que la gravité d'une maladie héréditaire peut être très variable entre membres d'une même famille. Cette variabilité du phénotype, conférée par des mutations identiques chez des patients différents, représente une des limitations majeures du conseil génétique. Cela est particulièrement vrai pour les prédispositions génétiques au cancer qui, pour se manifester, peuvent nécessiter des événements stochastiques tels que des mutations somatiques. Aussi importe-t-il de bien identifier et de délimiter le rôle des différents facteurs pouvant contribuer à déterminer l'expressivité d'une anomalie génétique. Une série récente d'articles s'attache à clarifier ces rapports dans le cas d'une maladie prédisposant au cancer colorectal, la polypose adénomateuse familiale (FAP).

La FAP est une maladie héréditaire autosomique dominante pouvant présenter trois groupes de manifestations.

- Des polypes adénomateux multiples dans le colon et le rectum, qui se développent rarement avant la puberté. Le nombre et l'âge d'apparition de ces tumeurs varient notablement d'un sujet à l'autre, si bien qu'en absence de test génétique, il est proposé, aux sujets nés d'un parent atteint, des coloscopies annuelles jusqu'à environ quarante ans, âge où l'expressivité de cette manifestation atteint un niveau proche de 1. Lorsque les polypes deviennent trop nombreux, est alors proposée une colectomie qui permet d'éliminer le principal risque tumoral, tout en préservant une bonne qualité de vie.

- Des signes oculaires dus à une hypertrophie de la couche pigmentaire de la rétine, qui se traduisent par des taches visibles au fond d'œil. Cette manifestation, précoce puisqu'elle peut être présente à la naissance, reste stable au cours de la vie. Des critères de nombre et de taille ont été définis, qui la rendent pathognomonique de la maladie. Son utilisation dans un but diagnostique est cependant limitée par son absence chez près de $30 \%$ des patients.

- Des manif estations extracoliques, essentiellement constituées de proliférations, parfois malignes, de différents tissus. Les plus remarquables, par leur gravité et leur fréquence élevées, sont les tumeurs duodénales et les tumeurs desmoïdes, pour la plupart de localisation intraabdominale, dont le développement, dans un contexte de polypose adénomateuse familiale, a été individualisé sous la dénomination de syndrome de Gardner dès 1953.

Le gène dont les altérations sont responsables de la FAP a été identifié en 1991. Son produit est une grosse protéine qui, en interagissant avec la $\beta$-caténine (et peut-être l' $\alpha$ caténine), intervient au niveau de la plaque des jonctions adhérentes $\left(\mathrm{m} / \mathrm{s}, n^{\circ} 2\right.$, vol. 10, p. $\left.228[1,2]\right)$. Ce site cellulaire particulier apparaît aujourd'hui comme un lieu privilégié de transmission de signaux réglant le cycle cellulaire. Le produit d'au moins un autre gène impliqué dans une prédisposition au développement de tumeurs, la neu- rofibromatose de type 2 , y réside vraisemblablement $[3,4]$. La quasitotalité des mutations ponctuelles du gène $A P C$ associées à une FAP a pour conséquence la synthèse d'une protéine tronquée; cette particularité a permis récemment de proposer des méthodes simplifiées de détection des mutations $A P C[5$, 6]. Plus rarement, son inactivation complète est provoquée par la délétion de la totalité de la séquence codante [7].

De nombreuses observations cliniques rapportent une variabilité des manif estations de la maladie entre membres atteints d'une même famille. Elles ont été complétées par des observations similaires chez des sujets génétiquement indépendants, porteurs cependant d'une mutation germinale identique du gène $A P C$. Une hypothèse permettant de rendre compte en partie de ces observations est basée sur la possible intervention du fond génétique dans le niveau d'expression des signes. La démonstration rigoureuse d'une telle hypothèse est difficile chez l'homme. Elle a été obtenue chez l'animal. En effet, un gène conditionne le nombre de tumeurs intestinales se développant chez des souris porteuses d'une mutation constitutionnelle sur le gène $A P C$. Il a été dénommé mom-1 et vient d'être localisé sur le chromosome 4 murin dans une région synténique avec la bande 1p35-36 humaine [8]. Cette région est fréquemment le site de pertes alléliques dans les tumeurs humaines, en particulier dans les tumeurs colorectales, ce qui conduit les auteurs à envisager que $M O M-1$ 


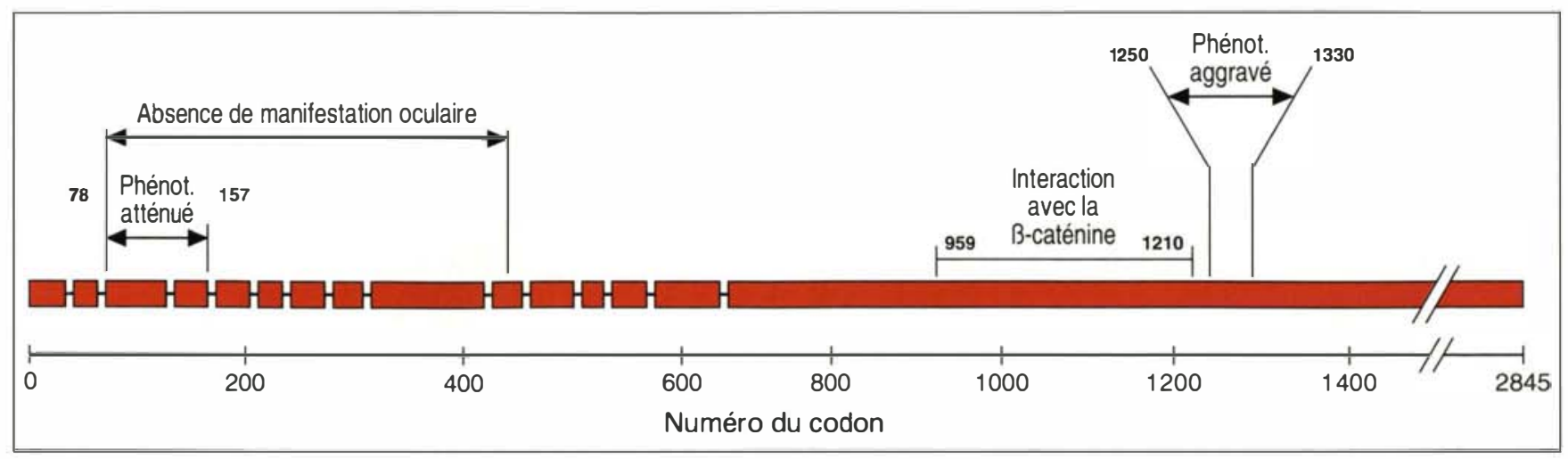

Figure 1. Le gène APC comporte 15 exons indiqués ici par des rectangles rouges. La quasi-totalité des mutations ponctuelles de la lignée germinale conduit à la synthèse d'une protéine tronquée. Le phénotype de la polypose adénomateuse présente des variations en fonction de la position de la mutation causale. Les mutations se trouvant entre les codons 78 et 157 confèrent un phénotype atténué. Celles survenant entre les codons 1250 et 1330 sont fréquentes et associées à une densité d'adénomes coliques élevée (phénotype aggravé). Lorsque la mutation survient avant l'exon 9, il n'y a pas de manifestation oculaire. Les très rares cas de mutations décrites après le codon 1597 sont mal caractérisés sur le plan phénotypique. Aucune mutation n'a été décrite avant le codon 78. La région de la protéine $A P C$ interagissant avec la $\beta$-caténine est aussi indiquée.

humain pourrait avoir un rôle suppresseur de tumeur.

Il semble également que la nature même de la mutation sur le gène $A P C$ puisse intervenir dans le niveau d'expression du phénotype. Cette intervention limiterait la variabilité intrafamiliale de l'expression phénotypique, et rendrait plus aisément compte d'une grande variabilité interfamiliale.

L'étude de trente-trois familles atteintes de FAP montre que la variabilité interfamiliale de l'expression des signes oculaires est significativement plus importante que la variabilité intrafamiliale. En réalité, une corrélation surprenante apparaît lorsque l'on prend en compte la position de la mutation le long de la séquence codante : les signes oculaires ne sont retrouvés que si la mutation survient après l'exon 9 . Cette observation fournit un cadre permettant de préciser l'intérêt et les limites de l'utilisation de l'examen ophtalmologique pour le diagnostic présymptomatique de la FAP [7].

La variabilité des manif estations coliques semble aussi être déterminée par la position des mutations. Les mutations dans l'exon 15 survenant entre les codons 1250 et 1464 (incluant le codon le plus fréquemment siège de mutations) sont associées à une densité d'adénomes sur la muqueuse colique deux à cinq fois supérieure à celle observée pour les mutations survenant dans les autres régions du gène [9]. Les mutations les plus proches du codon initiateur sont, au contraire, associées à un phénotype atténué, se révélant en général tardivement et sans manif estation extracolique, pour lequel une prise en charge thérapeutique spécifique doit sans doute être définie [10].

La gravité des manifestations de la FAP n'est pas uniquement déterminée génétiquement. Ainsi, il est possible que la variabilité intrafamiliale des signes coliques, qui est plus importante que celle des signes oculaires, soit en partie conditionnée par des facteurs environnementaux. Il a été rapporté que la prise d'antiinflammatoires non stéroïdiens [11] pourrait ralentir le développement de certains adénomes, suggérant la possibilité d'intervention médicamenteuse. Malheureusement ces médicaments ne semblent pas permettre d'éviter l'apparition de carcinomes [12].

A l'heure actuelle, nous ne connaissons pas suffisamment bien la struc- ture de la protéine, ni sa fonction, ni les modalités d'expression du gène $A P C$ pour fournir à ces différentes observations des explications basées sur un mécanisme moléculaire précis. L'hypothèse tentante que certaines formes tronquées de la protéine APC pourraient contribuer, soit à diminuer, soit à aggraver le phénotype doit bien sûr être maintenant examinée en développant des systèmes cellulaires ou animaux adaptés

\section{RÉFÉRENCES}

1. Rubinf eld B, Souza B, Albert I, Müller O, Chamberlain SH, Masiarz FR, Munemitsu S, Polakis P. Association of the APC gene product with $\beta$-catenin. Science 1993 ; 262: 1731-4.

2. Su LK, Vogelstein B, Kinzler KW. Association of the APC tumor suppressor protein with catenins. Science 1993; 262: 1734-7.

3. Rouleau G, Mérel P, Luchtman M, Sanson M, Zucman J, Marineau C, HoangXuan K, Demczuk S, Desmaze C, Plougastel B, Pulst SM, Bijlsma E, Fashold R, Dumanski J, de Jong $P$, Parry D, Eldrige R, Aurias A, Delattre O, Thomas G. Alteration in a new gene encoding a putative membrane organizing protein causes neurofibromatosis type 2 . Nature 1993; 363 : 515-21. 
4. Trof atter JA, MacCollin MM, Rutter JL, Murrell IR, Duyao MP, Parry D, Eldrige R, Kley N, Menon AG; Pulaski K, Haase VH, Ambrose CM, Munroe D, Bove (', Haines JL, Martuza RL, MacDonald ME, Seizinger BR, Short MP, Buckler AJ, Gusella JF. A novel moesin-, ezrin-, radixin-like gene is a candidate for the neurofibromatosis 2 tumor suppressor. Cell 1993; 72: 791-800.

5. Powell SM, Petersen GM, Krush AJ, Booker S, Jen J, Giardiello FM, Hamilton SR, Vogelstein B, Kinzler KW. Molecular diagnosis of familial adenomatous polvposis. $N$ Engl J Med 1993; 329 : 1982-7.

6. Varesco L, (Groclen J, Spirio L, el al. A rapid screening method to detect nonsense and frameshift mutations : identification of disease-causing APC: alleles. Cancer Res $1993 ; 53$ : 5581-4.

7. Olschwang S, Tiret A, Laurent-Puig P, Muleris M, Parc R, Thomas (;. Restriction of ocular fundus lesions to a specific subgroup of APC : mutations in adenomatous polyposis coli patients. Cell 1993; 75 : 959-68.

8. Dietrich WF, Iander ES, Smith JS, el al. (ienetic identification of mom-l, a major modifier locus affecting min-induced intestinal neoplasia in the mouse. Ciell 1993; 75: 631-9.

9. Nagase H, Miyoshi Y, Horii A, el al. Correlation between the location of germline mutations in the $A P^{\prime}($ g gene and the number of colorectal polyps in familial adenomatous polyposis patients. Ciancer Res 1992 ; 52: 4055-7.

10. Spirio L, Olschwang S, Groden J, el al. Alleles of the $A P(:$ gene: an attenuated form of familial polyposis. Cell 1993; 75 : 951-7.

11. Giardiello FM, Hamilton SR, Krush AJ, Piantadosi S, Hylind LM, Celano P, Booker SV, Robinson CR, Offerhaus G. Treatment of colonic and rectal aclenomas with sulindac in familial aclenomatous polyposis. $N$ Engl J Med 1993; 328: 1313-6.

12. Thorson AG; Lunch HT, Smyrk TC. Rectal cancer in FAP patient after sulindac. Lancel $1994 ; 343: 180$.

\section{Sylviane Olschwang Pierre Laurent-Puig \\ Gilles Thomas}

Institut Curie, 26, rue d'Ulm, 75231 Paris Cedex 05, France.

TIRÉS A PART

G. Thomas. 